\title{
Trajetórias de Habilidades Sociais e Problemas de Comportamento no Ensino Fundamental: Influência da Educação Infantil
}

\section{Trajectories of Social Skills and Behavior Problems in Primary School: Influence of Early Childhood Education}

\author{
Elaine Cristina Gardinal Pizato ${ }^{a}$, Edna Maria Marturano* ${ }^{*}$, \\ \& Anne Marie Germaine Victorine Fontaine ${ }^{c}$ \\ ${ }^{a}$ Faculdades Integradas de Jahu, Jaú, São Paulo, Brasil, \\ ${ }^{b}$ Universidade de São Paulo, Ribeirão Preto, São Paulo, Brasil \\ $\&{ }^{c}$ Universidade do Porto, Porto, Distrito do Porto, Portugal
}

\begin{abstract}
Resumo
A educação infantil - EI pode estar associada ao desenvolvimento psicossocial favorável de crianças durante o ensino fundamental - EF. Os objetivos deste estudo longitudinal foram: (a) investigar a estabilidade das habilidades sociais e dos comportamentos externalizantes e internalizantes das crianças na escola, do $3^{\circ}$ ao $5^{\circ}$ ano do EF; (b) investigar o curso das habilidades sociais e dos comportamentos problemáticos, em função da exposição à EI. Participaram 294 alunos do EF em cinco escolas públicas. As professoras responderam ao Social Skills Rating System no $3^{\circ}$, no $4^{\circ}$ e no $5^{\circ}$ ano. Controlado o nível socioeconômico, observou-se trajetória descendente de comportamentos problemáticos. A EI foi associada a menos problemas internalizantes e, entre as meninas, a melhores habilidades sociais. Palavras-chave: Habilidades sociais, problemas de comportamento, educação infantil, ensino fundamental.

Abstract

Early Childhood Education (ECE) may be associated with children's positive psychosocial development during Elementary School (ES). This longitudinal study has two aims: (a) to investigate the stability of children's social skills as well as externalizing and internalizing behaviors at school from $3^{\text {rd }}$ to $5^{\text {th }}$ year of ES; (b) to investigate the course of social skills and problem behaviors regarding the exposure to ECE. Participants were 294 ES students from five public schools. The teachers responded to the Social Skills Rating System in the $3^{\text {rd }}, 4^{\text {th }}$ and $5^{\text {th }}$ year. When socioeconomic status was controlled, behavior problems showed a descending trajectory. ECE was associated with fewer internalizing problems, and, among girls, with better social skills.

Keywords: Social skills, behavior problems, early childhood education, elementary school.
\end{abstract}

Este artigo focaliza trajetórias de desenvolvimento psicossocial de crianças no contexto escolar, durante o primeiro ciclo do ensino fundamental, levando em conta sua história prévia de exposição à educação infantil. Para investigar o desenvolvimento psicossocial, recorre-se aos constructos de habilidades sociais, comportamentos externalizantes e comportamentos internalizantes, representando distintos domínios do funcionamento adaptativo na infância (Bornstein, Hahn, \& Haynes, 2010; M. R. Reynolds, Sander, \& Irvin, 2010).

* Endereço para correspondência: Departamento de Neurociências e Ciências do Comportamento, Faculdade de Medicina de Ribeirão Preto, Universidade de São Paulo, Rua Tenente Catão Roxo, 2650, Ribeirão Preto, SP Brasil 14051-140. E-mail emmartur@fmrp.usp.br

Apoio: Fundação de Amparo à Pesquisa do Estado de São Paulo (FAPESP) e Conselho Nacional de Desenvolvimento Científico e Tecnológico (CNPQ).

\section{Domínios do Funcionamento Adaptativo na Infância}

O desenvolvimento psicossocial das crianças pode ser descrito em termos de competência e disfunção. A competência, como um indicador de adaptação positiva, mede o grau em que um indivíduo é capaz de negociar adaptativamente os desafios presentes. A competência é frequentemente operacionalizada por meio do constructo habilidades sociais (HS; ver, por exemplo, Sallquist et al., 2009). O conceito remete a um conjunto de comportamentos aprendidos, apresentados pelo indivíduo diante das demandas de uma situação interpessoal, que possibilitam sucesso em iniciar e manter interações sociais positivas e implicam em algum controle das próprias emoções (Berry \& O'Connor, 2010). Assertividade, solução de problemas interpessoais, cooperação, empatia, expressividade emocional e habilidades sociais acadêmicas são exemplos de classes de habilidades sociais (Bolsoni-Silva, Marturano, \& Freiria, 2010; Del Prette \& Del Prette, 2005). 
Durante os anos do ensino fundamental - EF, habilidades sociais contribuem para a aceitação no grupo de pares e para o aprendizado escolar (Blandon, Calkins, Grimm, Keane, \& O'Brien, 2010; Kettler, Elliott, Davies, \& Griffin, 2011). Ganhos no aprendizado são mediados em parte por efeitos de engajamento nas atividades escolares (Kettler et al., 2011). Diferenças de gênero e origem social têm sido relatadas; os professores em geral avaliam mais positivamente as meninas (Bandeira, Rocha, Freitas, Del Prette, \& Del Prette, 2006; Gardinal \& Marturano, 2007; Sallquist et al., 2009) e as crianças de nível socioeconômico (NSE) mais alto (Bandeira et al., 2006; M. R. Reynolds et al., 2010).

Expressões de disfunção, que se referem à manifestação recorrente de dificuldade em manter comportamentos de autocontrole, têm sido operacionalizadas mediante a noção de ajustamento comportamental, associado a dois fatores amplos. Comportamentos externalizantes incluem problemas com atenção e auto-regulação, bem como comportamentos anti-sociais e agressivos; comportamentos internalizantes incluem depressão, isolamento, ansiedade e queixas somáticas (Achenbach \& Edelbrock, 1979; Bornstein et al., 2010).

Problemas internalizantes e externalizantes têm sido associados a dificuldades no relacionamento com os pares e os externalizantes trazem maior risco para desfechos desfavoráveis, tais como envolvimento com pares desviantes e comportamentos de risco na adolescência (Fanti \& Henrich, 2010). Nos anos do EF, os dois tipos de problema apresentam correlação positiva entre eles, assim como associação negativa com HS (Berry \& O'Connor, 2010; Bornstein et al., 2010; M. R. Reynolds et al., 2010). Assim como nas HS, diferenças de gênero e origem social têm sido relatadas, principalmente para os comportamentos externalizantes, com maior risco para meninos (Miner \& Clarke-Stewart, 2008; Morgan, Farkas, \& Wu, 2009; Silver, Measelle, Armstrong, \& Essex, 2010) e crianças de menor NSE (Bohlin \& Hagekull, 2009; Dearing, McCartney, \& Taylor, 2006).

\section{Trajetórias de Desenvolvimento Psicossocial nos Anos do Ensino Fundamental}

Os anos do EF constituem um período de importantes conquistas desenvolvimentais. Na esfera do desenvolvimento psicossocial, há um declínio nos comportamentos disruptivos característicos da fase pré-escolar, à medida que as crianças desenvolvem estratégias mais eficazes de auto-regulação emocional, evoluindo para formas mais maduras de lidar com as demandas interpessoais emergentes (Bee, 2003; Skinner \& Zimmer-Gembeck, 2007).

Pesquisas sobre estabilidade das diferenças individuais em HS e problemas de comportamento, desde a educação infantil até os anos intermediários do EF, encontraram correlações moderadas entre escores obtidos em diferentes momentos do percurso escolar (Bolsoni-Silva et al., 2010; Sallquist et al., 2009), o que aponta para a modificabilidade dos comportamentos, tanto os socialmente habilidosos como os problemáticos (Bolsoni-Silva et al., 2010).
Estudos longitudinais com foco em trajetórias de desenvolvimento demonstram redução dos comportamentos externalizantes no EF (Bolsoni-Silva et al., 2010; Miner \& Clarke-Stewart, 2008; Robbers et al., 2010). Para comportamentos internalizantes, a evidência disponível é mais restrita. Com base na avaliação de professores, há indícios tanto de trajetória descendente, entre o jardim de infância e o $5^{\circ}$ ano do EF (Gazelle \& Ladd, 2003) como de trajetória ascendente no mesmo período (M. R. Reynolds et al., 2010).

As HS parecem seguir trajetórias de expansão, conforme indicado em estudos longitudinais (Berry \& O'Connor, 2010; Chen \& French, 2008; Miner \& Clarke-Stewart, 2008) e sugerido em pesquisa com desenho transversal (Bandeira et al., 2006). No entanto, algumas pesquisas têm trazido resultados diferentes. Sallquist et al. (2009), com base em quatro avaliações bianuais consecutivas, com crianças que na primeira coleta cursavam jardim de infância, $1^{\circ}, 2^{\circ}$ ou $3^{\circ}$ ano, encontraram modesta redução de comportamentos socialmente apropriados. No estudo de M. R. Reynolds et al. (2010), trajetórias de HS na escola, do jardim de infância ao $5^{\circ}$ ano, mostraram um decréscimo pequeno, mas significativo. Bolsoni-Silva et al. (2010), seguindo depois de quatro anos crianças avaliadas como socialmente habilidosas ou com problemas de comportamento na educação infantil - EI, verificaram aumento das HS, no EF, restrito ao grupo com problemas de comportamento, ao passo que no grupo socialmente habilidoso houve diminuição. Essas discrepâncias podem ser devidas a vários fatores, como, por exemplo, diferenças metodológicas entre as pesquisas. Por exemplo, no estudo de Berry e O'Connor, (2010) as mães avaliaram as HS das crianças e a relação professor-aluno fazia parte do modelo de predição, ao passo que na pesquisa de $\mathrm{M}$. R. Reynolds et al. (2010) as HS foram avaliadas pelos professores e as trajetórias de HS foram investigadas em um modelo composto que incluiu também trajetórias de internalização.

Dificuldades adaptativas precoces, expressas em altos níveis de comportamentos externalizantes ou mesmo internalizantes na escola, predizem trajetórias desfavoráveis (Morgan et al., 2009; Silver et al., 2010). Assim, há interesse em investigar o desenvolvimento psicossocial nos anos escolares, com base no repertório com que a criança ingressa no EF. Pesquisas com enfoque ecológico enfatizam preditores relacionais detectados já no contexto da EI. Por exemplo, há indícios de que aceitação ou rejeição pelos colegas, laços de amizade e exposição a estressores relacionais no jardim de infância podem ter efeitos persistentes na adaptação posterior (Silver et al., 2010).

\section{Educação Infantil e Desenvolvimento Psicossocial}

Dada a relevância de tais experiências precoces na escola, investimentos têm sido feitos para propiciar às crianças o acesso a programas pré-escolares que as instrumentem para os desafios do desenvolvimento. No Brasil, a EI tem como objetivo o desenvolvimento integral da 
Pizato, E. C. G., Marturano, E. M. \& Fontaine, A. M. G. V. (2014). Trajetórias de Habilidades Sociais e Problemas de Comportamento no Ensino Fundamental: Influência da Educação Infantil.

criança e constitui a primeira etapa da educação básica, abrangendo a creche para a faixa etária entre 0 e 3 anos e a pré-escola para a de 4 a 5 anos. A meta de universalização do acesso à pré-escola parece próxima, com $80 \%$ das crianças de 4 a 6 anos inseridas no sistema educacional em 2008, ainda que com importantes variações regionais (Vieira, 2010) e lacunas quanto à qualidade (Campos, Esposito, Bhering, Gimenes, \& Abuchaim, 2011). Nesse contexto, torna-se importante avaliar os efeitos da pré-escola sobre o desenvolvimento.

$\mathrm{O}$ acesso à pré-escola favorece o desenvolvimento cognitivo e psicossocial nos primeiros anos do ensino fundamental (Sylva, Melhuish, Sammons, Siraj-Blatchford, $\&$ Taggart, 2004). Efeitos protetores do desenvolvimento até a idade adulta, em populações de baixa renda, têm sido constatados para programas pré-escolares com rigoroso controle de qualidade (A. J. Reynolds \& Temple, 2008).

Estudos brasileiros pontuais relatam efeitos psicossociais positivos do acesso à EI logo no inicio do EF. Trivellato-Ferreira e Marturano (2008) constataram que crianças com pelo menos seis meses de EI, quando comparadas a crianças que não tinham tido acesso a esse nível de ensino, eram avaliadas pelo professor como preferidas pelos colegas de classe na $1^{a}$ série. A experiência na EI também parece atenuar sintomas de estresse relatados pela criança no início do EF: crianças sem pré-escola apresentaram índices maiores de sintomas de estresse, em comparação com as que tinham frequentado a EI (Trivellato-Ferreira \& Marturano, 2008). Além disso, a EI contribuiu para menor percepção de estresse no relacionamento com os colegas na $1^{\mathrm{a}}$ série (Marturano, Trivellato-Ferreira, \& Gardinal, 2009). Em estudo recente, alunos do $3^{\circ}$ ano com experiência de um ano em EI de escola pública mostraram mais $\mathrm{HS}$ e foram preferidos pelos colegas, quando comparados a crianças sem acesso prévio à EI (Pereira, Marturano, Gardinal-Pizato, \& Fontaine, 2011). Em que pesem os resultados positivos, faltam no país estudos sobre efeitos psicossociais da EI ao longo do EF.

Efeitos do tempo de exposição à EI sobre o desenvolvimento psicossocial têm sido menos explorados que os efeitos do acesso à EI. Os estudos disponíveis estão circunscritos a efeitos logo na entrada do EF. Na pesquisa relatada por Sylva et al. (2004), ingresso mais precoce na EI foi associado a melhor sociabilidade e autonomia no início do EF. Pereira et al. (2011) constataram que alunos do $3^{\circ}$ ano com dois anos de exposição à EI levavam vantagem sobre os colegas com um ano de exposição, em um indicador sociométrico de amizade baseado em escolhas recíprocas, mas não em medidas de HS.

\section{O Presente Estudo}

Os resultados dos estudos revisados brevemente nos parágrafos precedentes mostram algumas tendências: estabilidade moderada dos indicadores de competência e disfunção nos anos do EF; declínio dos comportamentos externalizantes e expansão das HS, com alguns resultados divergentes em relação às HS; impacto positivo do acesso à EI sobre o desenvolvimento psicossocial no limiar do EF. Efeitos psicossociais da EI que ultrapassam o início do EF têm sido escassamente explorados.

Nesse contexto, a pesquisa relatada neste artigo focalizou duas questões. A primeira diz respeito à estabilidade dos constructos selecionados para investigar o desenvolvimento psicossocial, examinada de dois modos: (a) continuidade diferencial, ou grau em que diferenças individuais permanecem estáveis no tempo; (b) estabilidade do nível médio, ou grau em que os escores médios de um constructo mudam através do tempo, como uma tendência desenvolvimental (Sallquist et al., 2009). A segunda questão explorada é a da influência da EI sobre trajetórias de HS e problemas de comportamento no EF, em anos escolares posteriores ao ingresso nesse nível de ensino, bem como os possíveis efeitos moderadores do gênero nessas relações.

Foram objetivos da pesquisa: (a) investigar a estabilidade das HS e dos comportamentos externalizantes e internalizantes exibidos pelas crianças na escola, do $3^{\circ}$ ao $5^{\circ}$ ano do ensino fundamental; (b) investigar a trajetória de desenvolvimento das HS e dos comportamentos problemáticos, em função da exposição à EI. Dado que os professores atribuem mais HS e menos problemas de comportamento às meninas e às crianças de NSE mais alto, o sexo e o NSE foram considerados no desenho do estudo de modo a averiguar a influência desses fatores nas trajetórias desenvolvimentais.

\section{Método}

\section{Local e Participantes}

A pesquisa foi conduzida nas cinco escolas públicas de uma cidade do estado de São Paulo com aproximadamente 24.000 habitantes. De um total inicial de 351 crianças elegíveis para o estudo, participaram efetivamente 294 (146 meninos e 148 meninas), com idade entre 7 e 9 anos ( $M=100,94$ meses; $D P=4,58$ meses). Esses alunos frequentavam o $3^{\circ}$ ano do ensino fundamental em $2007 \mathrm{e}$ foram seguidos até 2009 , quando no $5^{\circ}$ ano. Trinta alunos não tinham frequentado a EI, 78 haviam completado um ano na EI e 186 haviam concluído dois anos na EI. Essa amostra corresponde a $79 \%$ da população escolar do $3^{\circ}$ ano na rede pública do município em 2007, excluídos os alunos que não atendiam ao critério etário, seis cujos pais não consentiram com sua participação e 51 que durante a pesquisa se mudaram para outra cidade. Também participaram da pesquisa 37 professores, como informantes, sendo 12 em 2007, 13 em 2008 e 12 em 2009.

\section{Instrumentos}

O nível socioeconômico - NSE foi avaliado por meio do Critério Brasil (Associação Brasileira de Empresas de Pesquisa [ABEP], 2003). A avaliação das HS e dos comportamentos externalizantes e internalizantes foi realizada com o Sistema de Avaliação de Habilidades Sociais, versão brasileira (SSRS-BR; Bandeira, Del Prette, Del Prette, \& 
Magalhães, 2009), nos três anos de coleta de dados. Segue uma descrição dos instrumentos.

Critério de Classificação Econômica Brasil. É composto por onze itens: nove que investigam o número de bens de consumo duráveis da família, um que investiga o grau de instrução do chefe da família e um que avalia o número de empregados mensalistas na casa. Permite a estratificação das famílias em cinco classes, que neste estudo foram agrupadas em três: alta (classes A e B), média (classe C) e baixa (classes D e E).

Sistema de Avaliação de Habilidades Sociais (SSRS-BR). O sistema compreende três escalas: HS, problemas de comportamento e competência acadêmica. Foram utilizadas as escalas de HS e problemas de comportamento da versão para o professor. Bandeira et al. (2009) reportam índices satisfatórios de consistência interna e estabilidade temporal para esta versão do instrumento. Na adaptação brasileira, a escala de problemas de comportamento compreende os fatores comportamentos externalizantes e comportamentos internalizantes.

\section{Procedimentos}

A pesquisa foi aprovada pelo Comitê de Ética em Pesquisa - Faculdade de Filosofia, Ciências e Letras de Ribeirão Preto (FFCLRP; proc. n 306/2007-2007.1.335.59.0). Foi obtido o consentimento dos pais para a participação de seus filhos e o dos professores para participarem como informantes, mediante assinatura de Termo de Consentimento Livre e Esclarecido. As crianças deram seu consentimento verbal para participar.

Segundo o delineamento longitudinal da pesquisa, as crianças foram avaliadas por seus professores no segundo semestre do $3^{\circ}$ ano (agosto-setembro), no segundo semestre do $4^{\circ}$ ano (agosto-setembro) e no primeiro semestre do $5^{\circ}$ ano (maio-junho). No $3^{\circ}$ ano, os pais ou responsáveis preencheram o formulário do Critério de Classificação Econômica Brasil, durante reuniões de pais nas escolas, e os professores preencheram o SRSS-BR. Nos anos subsequentes, os professores preencheram o SSRS-BR.

\section{Análise Estatística}

A estabilidade das diferenças inter-individuais, bem como das relações entre variáveis comportamentais, foi investigada por meio do coeficiente de correlação de Pearson. $\mathrm{Na}$ etapa seguinte da análise, foram avaliados no mesmo modelo os efeitos da EI e da passagem dos anos no ensino fundamental sobre os indicadores de HS e problemas de comportamento. Essa análise foi precedida por uma série de sondagens. Para averiguar (a) a necessidade de controlar a influência eventual do NSE sobre as variáveis dependentes, (b) a pertinência da distinção entre anos de exposição à educação infantil e (c) a presença de diferenças de sexo, já clássicas na literatura, diferenças entre médias foram analisadas, por meio do teste $t$ de Student ou análise de variância, tendo como fatores de segmentação o NSE, o tempo de exposição à EI e o sexo. Os resultados das comparações entre médias foram levados em conta para a formulação do modelo subsequente que explorou a variação, com o tempo, do nível médio das HS e dos problemas de comportamento, em função da exposição à EI. No caso de serem observadas diferenças significativas, testes post-hoc foram conduzidos para identificar os fatores responsáveis por essas diferenças. Utilizou-se um nível alfa de 0,05 .

\section{Resultados}

As correlações entre as variáveis dependentes do estudo são apresentadas na Tabela 1, por sexo. Os resultados dos meninos se encontram acima da diagonal da tabela e os das meninas, abaixo da diagonal.

Tabela 1

Intercorrelações entre as Escalas nos Três Momentos da Avaliação, por Sexo

\begin{tabular}{|c|c|c|c|c|c|c|c|c|c|}
\hline & \multicolumn{3}{|c|}{ Habilidades sociais } & \multicolumn{3}{|c|}{$\begin{array}{c}\text { Comportamentos } \\
\text { externalizantes }\end{array}$} & \multicolumn{3}{|c|}{$\begin{array}{c}\text { Comportamentos } \\
\text { internalizantes }\end{array}$} \\
\hline & $3^{\circ}$ ano & $4^{\circ}$ ano & $5^{\circ}$ ano & $3^{\circ}$ ano & $4^{\circ}$ ano & $5^{\circ}$ ano & $3^{\circ}$ ano & $4^{\circ}$ ano & $5^{\circ}$ ano \\
\hline Habilidades sociais $3^{\circ}$ ano & - & $0,39 * *$ & $0,42 * *$ & $-0,55 * *$ & $-0,33 * *$ & $-0,32 * *$ & $-0,46^{* *}$ & $-0,36 * *$ & $-0,31 * *$ \\
\hline Habilidades sociais $4^{\circ}$ ano & $0,24 * *$ & - & $0,27 * *$ & $-0,34 * *$ & $-0,57 * *$ & $-0,25 * *$ & $-0,33 * *$ & $-0,57 * *$ & $-0,34 * *$ \\
\hline Habilidades sociais $5^{\circ}$ ano & $0,50 * *$ & $0,36^{* *}$ & - & $-0,32 * *$ & $-0,33 * *$ & $-0,66 * *$ & $-0,32 * *$ & $-0,27 * *$ & $-0,54 * *$ \\
\hline Externalizantes $3^{\circ}$ ano & $-0,42 * *$ & $-0,30 * *$ & $-0,35 * *$ & - & $0,46^{* *}$ & $0,39 * *$ & $0,54 * *$ & 0,14 & 0,13 \\
\hline Externalizantes $4^{\circ}$ ano & $-0,29 * *$ & $-0,50 * *$ & $-0,44 * *$ & $0,38 * *$ & - & $0,51 * *$ & $0,21 * *$ & $0,40 * *$ & 0,16 \\
\hline Externalizantes $5^{\circ}$ ano & $-0,42 * *$ & $-0,25^{* *}$ & $-0,71 * *$ & $0,33 * *$ & $0,61 * *$ & - & $0,16^{*}$ & 0,14 & $0,29 * *$ \\
\hline Internalizantes $3^{\circ}$ ano & $-0,41 * *$ & $-0,39 * *$ & $-0,38 * *$ & $0,57 * *$ & $0,20 *$ & $0,26 * *$ & - & $0,27^{* *}$ & $0,34 * *$ \\
\hline Internalizantes $4^{\circ}$ ano & $-0,14$ & $-0,57 * *$ & $-0,27 * *$ & $0,19 *$ & $0,53 * *$ & $0,28 * *$ & $0,24 * *$ & - & $0,35 * *$ \\
\hline Internalizantes $5^{\circ}$ ano &,$- 30 * *$ & $-0,34 * *$ & $-0,70 * *$ & $0,21 * *$ & $0,46^{* *}$ & $0,63 * *$ & $0,30 * *$ & $0,39 * *$ & - \\
\hline
\end{tabular}

Nota. Correlações para meninos acima da diagonal, para meninas abaixo da diagonal.

$* p<0,05 ; * *<0,01$. 
Pizato, E. C. G., Marturano, E. M. \& Fontaine, A. M. G. V. (2014). Trajetórias de Habilidades Sociais e Problemas de Comportamento no Ensino Fundamental: Influência da Educação Infantil.

As correlações entre medidas da mesma variável em diferentes momentos do percurso escolar são positivas e significativas, indicando estabilidade baixa ou moderada (correlações entre 0,24 e 0,61). Para meninos e meninas, maiores correlações ocorrem entre comportamentos externalizantes avaliados no $4^{\circ}$ e no $5^{\circ}$ ano. As correlações são baixas entre medidas de comportamentos internalizantes. Para as HS, as maiores correlações se verificam entre o $3^{\circ}$ e o $5^{\circ}$ ano, tanto para meninas como para meninos.

As correlações entre variáveis avaliadas no mesmo ano escolar são maiores que as correlações entre medidas da mesma variável em diferentes momentos, o que pode ser atribuído, pelo menos em parte, ao fato de as avaliações

Tabela 2 concorrentes do aluno terem sido feitas pelo mesmo professor, ao passo que as avaliações consecutivas foram feitas por diferentes professores. As correlações negativas entre HS e problemas de comportamento tendem a aumentar ao longo dos três anos, sendo essa tendência mais perceptível para as meninas, tanto para os comportamentos externalizantes como internalizantes. As correlações positivas entre comportamentos externalizantes e internalizantes mostram diminuição para os meninos a cada ano (de 0,54 para 0,29 ), ao passo que para as meninas observa-se um ligeiro aumento no $5^{\circ}$ ano.

Os resultados da ANOVA e do teste $t$ são apresentados na Tabela 2. Na tabela se encontram também as médias e os desvios-padrão das variáveis do estudo.

Médias e Desvios-Padrão das Variáveis do Estudo, por Grupos

\begin{tabular}{|c|c|c|c|c|c|c|c|c|}
\hline & \multicolumn{2}{|c|}{ Sexo } & \multicolumn{3}{|c|}{ Anos na educação infantil } & \multicolumn{3}{|c|}{ Classe socioeconômica } \\
\hline & $\begin{array}{l}\text { Meninos } \\
(n: 146)\end{array}$ & $\begin{array}{l}\text { Meninas } \\
(n: 148)\end{array}$ & $\begin{array}{c}0 \\
(n: 30)\end{array}$ & $\begin{array}{c}1 \\
(n: 78)\end{array}$ & $\begin{array}{c}2 \\
(n: 186)\end{array}$ & $\begin{array}{c}\text { Alta } \\
(n: 63)\end{array}$ & $\begin{array}{l}\text { Média } \\
(n: 143)\end{array}$ & $\begin{array}{l}\text { Baixa } \\
(n: 88)\end{array}$ \\
\hline Habilidades sociais $3^{023}$ & $45,9(13,8)$ & $49,0(12,5)$ & $40,2(11,7)$ & $47,7(11,1)$ & $48,6(14,0)$ & $52,9(13,15)$ & $47,1(12,9)$ & $44,2(12,7)$ \\
\hline Habilidades sociais $4^{\circ} 123$ & $47,0(15,4)$ & $52,7(13,2)$ & $41,7(13,9)$ & $48,6(13,6)$ & $51,7(14,6)$ & $57,1(11,3)$ & $49,2(14,6)$ & $45,6(14,8$ \\
\hline Habilidades sociais $5^{\circ 123}$ & $51,5(13,9)$ & $55,1(14,8)$ & $43,2(16,5)$ & $54,9(13,4)$ & $54,3(14,0)$ & $60,5(9,11)$ & $53,5(14,8)$ & $48,0(14,9)$ \\
\hline Externalizantes $3^{\circ} 13$ & $9,3(6,9)$ & $6,1(6,6)$ & $10,4(7,8)$ & $7,3(6,4)$ & $7,5(7,0)$ & $4,9(6,2)$ & $7,7(7,0)$ & $9,7(6,9)$ \\
\hline Externalizantes $4^{\circ} 13$ & $6,9(6,5)$ & $5,0(6,2)$ & $7,8(6,3)$ & $5,5(6,4)$ & $5,9(6,4)$ & $3,6(4,8)$ & $6,6(6,8)$ & $6,8(6,4)$ \\
\hline Externalizantes $5^{\circ} 13$ & $5,8(5,7)$ & $3,7(5,5)$ & $6,0(5,2)$ & $3,5(4,7)$ & $5,0(6,1)$ & $2,7(4,4)$ & $4,9(5,8)$ & $5,9(6,0)$ \\
\hline Internalizantes $3^{\circ} 123$ & $4,1(3,3)$ & $3,4(3,1)$ & $5,5(3,6)$ & $3,7(3,2)$ & $3,5(3,1)$ & $2,7(3,0)$ & $3,7(3,0)$ & $4,5(3,4)$ \\
\hline Internalizantes $4^{\circ} 123$ & $3,3(3,0)$ & $2,6(2,6)$ & $4,7(3,1)$ & $2,9(2,6)$ & $2,7(2,8)$ & $1,7(2,2)$ & $3,2(2,9)$ & $3,4(3,0)$ \\
\hline Internalizantes $5^{\circ} 23$ & $2,3(2,6)$ & $2,0(2,6)$ & $3,7(2,8)$ & $1,6(2,1)$ & $2,2(2,6)$ & $1,2(1,6)$ & $2,3(2,5)$ & $2,7(3,1)$ \\
\hline
\end{tabular}

Nota. Diferença estatisticamente significativa $(p<0,05)$ entre grupos: ${ }^{1}$ para sexo; ${ }^{2}$ para anos na educação infantil; ${ }^{3}$ para classe socioeconômica.

A classificação socioeconômica foi associada positivamente a HS e negativamente a comportamentos problemáticos em todas as comparações. Foram obtidos os seguintes resultados para HS: $3^{\circ}$ ano, $F_{2,291}=8,44, p$ $<0,001 ; 4^{\circ}$ ano, $F_{2291}=12,47, p<0,001 ; 5^{\circ}$ ano, $\mathrm{F}_{2,291}=$ $15,02, \mathrm{p}<0,001$. Para comportamentos externalizantes: $3^{\circ}$ ano, $F_{2,291}=9,34, p<0,001 ; 4^{\circ}$ ano, $F_{2,291}=5,71, p<$ 0,$01 ; 5^{\circ}$ ano, $F_{2,291}=5,97, p<0,01$. Para comportamentos internalizantes: $3^{\circ}$ ano, $F_{2291}=6,65, p<0,01,4^{\circ}$ ano $F_{2291}$ $=7,77, p<0,01,5^{\circ}$ ano, $F_{2291}=7,33, p<0,01$.

A exposição à EI foi associada às HS em todos os momentos; no $3^{\circ}$ ano $\left(F_{2,291}=5,36, p<0,01\right)$, no $4^{\circ}$ ano $\left(F_{2}\right.$ $\left.{ }_{291}=6,74, p<0,01\right) \mathrm{e}$ no $5^{\circ}$ ano $\left(F_{2291}=8,65, p<0,001\right)$. Testes post-hoc indicaram, em todas as comparações, que apenas o grupo sem acesso à EI se diferencia, com médias significativamente menores que os demais.

Para comportamentos externalizantes não foram detectadas diferenças significativas associadas ao tempo de exposição à EI, mas sim tendências, no $3^{\circ}\left(F_{2,291}=2,51\right.$, $p=0,08)$ e no $5^{\circ}$ ano $\left(F_{2,291}=2,65, p=0,07\right)$. Para comportamentos internalizantes foram detectadas diferenças no $3^{\circ}\left(F_{2,291}=5,36, p<0,01\right)$, no $4^{\circ}\left(F_{2,291}=6,93, p<\right.$ $0,01)$ e no $5^{\circ}$ ano $\left(F_{2,291}=7,45, p<0,01\right)$. Os testes post-hoc indicaram que o grupo sem acesso à EI teve médias significativamente maiores em relação aos dois grupos com passagem pela EI.

Foram detectadas diversas diferenças entre os sexos. As meninas foram avaliadas como socialmente mais habilidosas pelos professores do $4^{\circ}\left(t_{294}=3,10, p<0,01\right)$ e do $5^{\circ}$ ano $\left(t_{294}=2,15, p<0,05\right)$. Já os meninos foram avaliados com mais comportamentos externalizantes no $3^{\circ}\left(t_{294}=4,03, p\right.$ $<0,001)$, no $4^{\circ}\left(t_{294}=2,56, p<0,05\right)$ e no $5^{\circ}$ ano $\left(t_{294}=\right.$ $2,15, p<0,05)$. Também em relação aos comportamentos internalizantes os meninos tiveram médias maiores, no $3^{\circ}$ $\left(t_{294}=1,98, p<0,05\right)$ e no $4^{\circ}$ ano $\left(t_{294}=2,24, p<0,05\right)$.

Os resultados apresentados na Tabela 2 indicam diferenças associadas ao sexo e ao NSE. Para o tempo de exposição à EI, apenas o grupo sem acesso obteve 
médias inferiores aos demais. Tais resultados justificam a opção em, por um lado, não discriminar, nas análises subsequentes, o tempo de exposição à EI, e por outro controlar os efeitos do NSE e do sexo, colocando-os como covariadas. Para avaliar o efeito do tempo de escolaridade e da frequência à EI nas habilidades e comportamentos das crianças durante o $\mathrm{EF}$, análises de variância com medidas repetidas foram processadas, tendo como variável independente intergrupo o acesso à $E I$ (sem acesso $\mathrm{x}$ com acesso), como variável independente intra-sujeito o tempo no ensino fundamental $\left(3^{\circ}, 4^{\circ}\right.$ e $5^{\circ}$ ano), como variáveis dependentes as HS, comportamentos externalizantes e internalizantes e como variáveis covariadas o NSE (três classes) e o sexo.

Para as HS, não se observou efeito da passagem do tempo, mas efeito significativo associado à EI: $F_{1,292}=$ $15,10, p<0,001$. Não houve efeitos de interação. Em relação aos comportamentos externalizantes, os resultados indicaram efeito significativo do tempo: $F_{1,292}=13,74, p$ $<0,001$. As comparações post hoc mostraram redução de comportamentos externalizantes, ano a ano, no decorrer do EF. Não houve efeito associado à EI. Um efeito de interação sexo x EI indicou que o acesso à EI está associado a menos manifestações externalizantes nas meninas: $F_{1,292}$ $=4,513, p<0,05$. Quanto aos comportamentos internalizantes, houve diminuição no decorrer do EF: $F_{1,292}=$ $9,24, p<0,01$. Nas comparações post hoc observaram-se diferenças significativas entre todos os anos. De acordo com a análise realizada intergrupos, houve um efeito significativo do acesso à EI, $F_{1,292}=16,40, p<0,001$. Não foram encontrados efeitos de interação.

Para uma identificação mais precisa dos efeitos diferenciais da EI nos meninos e nas meninas, foram conduzidas novas análises separadamente para cada sexo. Enquanto na amostra masculina não se detectam efeitos da passagem do tempo ou da EI para as HS, na amostra feminina há efeito significativo do acesso à EI $\left(F_{1,146}=15,32, p<0,001\right)$. Pode-se inferir que os resultados da análise anterior para o grupo total seriam essencialmente devidos às características da amostra feminina. Ainda na amostra feminina, um efeito de interação foi detectado entre tempo e NSE. Para esclarecer esse resultado, análises de variância com medidas repetidas foram processadas para cada uma das três classes econômicas - alta, média e baixa. Apenas na classe média, houve aumento das HS, relacionado ao avanço na escolaridade $\left(F_{2,65}=6,36, p<0,05\right)$. Comparações post hoc indicaram diferença entre a média do $3^{\circ}$ ano, 43,24 e a média do $5^{\circ}$ ano, igual a 49,99 .

Não se observou efeito do tempo sobre os comportamentos externalizantes das meninas. Para os meninos houve redução $\left(F_{2,143}=16,25, p<0,001\right)$ entre o $3^{\circ}$ e o $5^{\circ}$ ano, de acordo com as comparações post hoc. Houve efeito do acesso à $\operatorname{EI}\left(F_{1,146}=6,94, p<0,01\right)$ nos comportamentos externalizantes das meninas, mas não dos meninos, o que permite melhor compreender os efeitos de interação na amostra global.
Quanto aos comportamentos internalizantes, houve efeito significativo do tempo para meninas $\left(F_{2,145}=4,50\right.$, $p<0,05)$ e meninos $\left(F_{2,143}=6,14, p<0,05\right)$. Nas comparações post hoc das meninas observou-se redução entre 0 $3^{\circ}$ e o $4^{\circ}$ ano e entre o $3^{\circ}$ e o $5^{\circ}$ ano. Para os meninos as comparações post hoc não mostraram redução entre o $3^{\circ} \mathrm{e}$ o $4^{\circ}$ ano, mas entre estes e o $5^{\circ}$ ano. Também se observou efeito significativo da EI, tanto nas meninas $\left(F_{1,146}=14,78\right.$, $p<0,001)$, como nos meninos $\left(F_{1,144}=4,35, p<0,05\right)$.

\section{Discussão}

Neste estudo se explorou o curso do desenvolvimento psicossocial no primeiro ciclo do EF, assim como a influência da EI nesse desenvolvimento, tal como operacionalizado por meio dos constructos HS, comportamentos externalizantes e comportamentos internalizantes, avaliados pelos professores. Nas análises preliminares, constataram-se variações nas medidas de interesse, associadas a sexo, nível socioeconômico e acesso à EI. Não foram detectadas variações associadas ao tempo de exposição à EI, medido em anos.

Diferenças de sexo aparecem a partir do $4^{\circ}$ ano para as HS e os comportamentos externalizantes. Elas são consistentes com achados de estudos prévios, em que professores atribuíram mais HS e menos comportamentos externalizantes às meninas (Bandeira et al., 2006; Miner \& Clarke-Stewart, 2008; Morgan et al., 2009; Sallquist et al., 2009; Silver et al., 2010). As HS mais desenvolvidas das meninas podem, no meio escolar, favorecer as relações entre pares e estimular a função de suporte social do grupo de pares em situação de estresse (Blandon et al., 2010). Os meninos foram avaliados com mais problemas internalizantes no $3^{\circ}$ e no $4^{\circ}$ ano, o que também está de acordo com a (escassa) literatura disponível, sugerindo que os meninos estão em maior risco para problemas internalizantes nos anos iniciais do ensino fundamental (Morgan et al., 2009).

Resultados referentes ao efeito do NSE apareceram em todos os momentos de avaliação, afetando os três indicadores de funcionamento psicossocial. Em concordância com a literatura, melhor condição socioeconômica foi associada a mais HS (Bandeira et al., 2006; M. R. Reynolds et al., 2010), assim como a menos comportamentos externalizantes e internalizantes (Bohlin \& Hagekull, 2009; Dearing et al., 2006). A influência do NSE está relacionada à escolaridade dos pais e poderia ser explicada por uma diversidade de processos familiares, com destaque para práticas educativas e sensibilidade materna, afetando tanto as HS como os problemas de comportamento (Brownell et al., 2008; Côté, Vaillancourt, Barker, Nagin, \& Tremblay, 2007). Esse resultado tem implicações para políticas públicas voltadas à infância; sabe-se, por exemplo, que cuidados alternativos de qualidade têm efeito protetor contra problemas externalizantes em crianças de NSE baixo (Bohlin \& Hagekull, 2009). 
Pizato, E. C. G., Marturano, E. M. \& Fontaine, A. M. G. V. (2014). Trajetórias de Habilidades Sociais e Problemas de Comportamento no Ensino Fundamental: Influência da Educação Infantil.

Nessas análises preliminares também foram detectados efeitos do acesso à EI, em que pese o fato de apenas $10 \%$ da amostra aproximadamente não ter frequentado a pré-escola; porém não se encontrou efeito do tempo de exposição à EI, mensurado em anos. Nos três momentos de avaliação, o acesso à EI foi associado a HS mais desenvolvidas e menos comportamentos internalizantes. Esses resultados corroboram achados obtidos com crianças mais jovens, indicativos de maior competência social e menos sintomas de estresse no $1^{\circ}$ ano do EF, para crianças com acesso prévio à EI (Trivellato-Ferreira \& Marturano, 2008). Eles replicam resultados de Pereira et al. (2011) sobre HS em alunos do $3^{\circ}$ ano.

As trajetórias de desenvolvimento foram investigadas quanto à estabilidade das diferenças individuais e quanto a tendências desenvolvimentais modais. Em relação à estabilidade, correlações baixas ou moderadas foram encontradas entre avaliações consecutivas de HS, assim como de comportamentos externalizantes. Já os comportamentos internalizantes mostraram baixa continuidade das diferenças individuais.

Estabilidade moderada para HS nos anos do EF já haviam sido relatadas (Sallquist et al., 2009). Recentemente, Bolsoni-Silva et al. (2010) encontraram correlações moderadas entre medidas de HS obtidas na EI e no $\mathrm{EF}$, com quatro anos de intervalo entre as avaliações. Essas autoras também obtiveram indícios de estabilidade moderada para comportamentos externalizantes, porém apenas para crianças com problemas de comportamento na EI e não para crianças consideradas pelos professores da EI como socialmente habilidosas. Como sugerem as autoras, estabilidade moderada pode ser vista como sinal de maleabilidade, de suscetibilidade a influências ambientais, o que favorece intervenções na escola, para promoção de HS e prevenção de problemas de comportamento.

Tendências desenvolvimentais relacionadas ao curso do desenvolvimento das HS e dos comportamentos externalizantes e internalizantes foram examinadas como função do acesso à EI. No exame dessas associações, o impacto do NSE foi controlado e foram analisados mais pormenorizadamente os efeitos do sexo, fatores que foram associados às variáveis dependentes nas análises preliminares.

Para as HS, não se observou variação no tempo na amostra total e no grupo masculino. No grupo feminino, observou-se aumento nas HS, entre o $3^{\circ}$ e o $5^{\circ}$ ano, mas apenas para crianças de NSE médio. A literatura apresenta resultados díspares sobre tendências modais de desenvolvimento das HS durante os anos do EF. Há relatos de ampliação (Berry \& O'Connor , 2010; Chen \& French, 2008; Miner \& Clarke-Stewart, 2008), como de redução (M. R. Reynolds et al., 2010; Sallquist et al., 2009). Conforme assinalado na introdução, diferenças metodológicas entre os estudos podem ser evocadas para explicar as divergências. No entanto, pode-se também especular que, como as HS são aprendidas, as discrepâncias encontradas reflitam parcialmente a diversidade de oportunidades para esse aprendizado nos ambientes de desenvolvimento, particularmente nas interações com os pares (Brownell et al., 2008).

Para comportamentos externalizantes, diferenças significativas entre todos os momentos foram detectadas na amostra total. A diminuição encontrada tem amplo respaldo na literatura (Bolsoni-Silva et al., 2010; Miner \& Clarke-Stewart, 2008; Robbers et al., 2010) e corresponde a uma tendência de desenvolvimento, relacionada ao aumento da auto-regulação (Bee, 2003; Skinner \& Zimmer-Gembeck, 2007). O resultado se repetiu no grupo dos meninos, especificamente entre o $3^{\circ}$ e o $5^{\circ}$ ano.

Os comportamentos internalizantes mostraram declínio, com diferenças significativas entre todos os momentos de avaliação. Essa foi uma tendência forte nos resultados, pois apareceu na amostra total, no grupo masculino e no grupo feminino. Em comparação a outros estudos na mesma faixa etária, os resultados confirmam aqueles obtidos por Gazelle e Ladd (2003), mas se contrapõem aos da pesquisa recente de M. R. Reynolds et al. (2010), que encontraram discreto aumento de sintomas internalizantes no SSRS, do jardim de infância ao $5^{\circ}$ ano. Cabe considerar que M. R. Reynolds et al. (2010) empregaram um modelo de análise diferente do nosso, focalizando trajetórias conjuntas de internalização e HS.

Os resultados encontrados, de diminuição dos sintomas internalizantes no primeiro ciclo do $\mathrm{EF}$, são compatíveis com a perspectiva de transição escolar. De acordo com essa perspectiva, o ingresso no ensino fundamental seria uma condição geradora de estresse e ansiedade, devido à imprevisibilidade / incontrolabilidade da nova condição (Marturano et al., 2009; Trivellato-Ferreira \& Marturano, 2008). Ao longo do tempo, com a formação de laços de amizade, o apoio dos pares e a familiarização ao contexto escolar, estável e estruturado, as crianças tenderiam a se sentir mais seguras, diminuindo assim a ansiedade.

Em suma, os resultados relativos ao curso do desenvolvimento apontaram clara diminuição dos problemas emocionais e de comportamento, assim como, em meninas de classe média, aumento das HS. Nem todos os resultados encontrados têm apoio na literatura. $\mathrm{Na}$ apreciação das discrepâncias, é preciso lembrar que diferentes estudos usaram modelos estatísticos diferentes e incluíram em seus modelos variáveis preditoras também diferentes, conforme exemplificado na introdução.

A propósito, pesquisas que investigaram relações entre os diferentes domínios adaptativos no curso do desenvolvimento mostram intricadas influências transacionais entre competência social, problemas externalizantes e problemas internalizantes (Bornstein et al., 2010; Fanti \& Henrich, 2010). Ainda que de forma limitada, influências transacionais foram captadas no presente estudo, por meio das correlações negativas progressivamente maiores entre domínios, à medida que as crianças avançam no EF. Tais correlações sugerem o mesmo efeito protetor das HS identificado por Bornstein et al. (2010). No presente estudo, 
esse efeito parece dar-se sobre os comportamentos externalizantes para ambos os sexos, e sobre os internalizantes para as meninas.

Efeitos do acesso à EI, apontados nas sondagens preliminares, foram confirmados nas análises de variância com medidas repetidas, tanto para a amostra total, incluindo o NSE e o sexo como covariadas, quanto nas amostras de meninos e meninas, mantido o NSE como covariada. $\mathrm{Na}$ amostra total, o acesso à EI implicou em HS mais desenvolvidas e menos comportamentos internalizantes no EF. Esses resultados se mantiveram nos três anos escolares em que as crianças foram acompanhadas, apesar de o percentual de alunos sem acesso à EI ser pouco mais de $10 \%$ da amostra. Reitera-se assim que o acesso à pré-escola pode favorecer o desenvolvimento psicossocial nos primeiros anos do EF (Sylva et al., 2004).

Pode-se supor que a passagem pela EI afeta o desenvolvimento das HS por meio da participação em brincadeiras, que oferecem oportunidade para as crianças lidarem com conflitos e aprenderem habilidades de auto-regulação, resolução de problemas, tomada de perspectiva e regras sociais, entre outras (Brownell et al., 2008). Na mesma direção se pode tentar entender o efeito protetor da EI, no que se refere aos sintomas internalizantes: as crianças com passagem pela EI terão mais recursos para lidar com demandas interpessoais e por isso se sentirão menos apreensivas no novo contexto (Marturano et al., 2009).

Os resultados das análises processadas por sexo, mantido o NSE como covariada, evidenciaram tendências diferentes para meninos e meninas. $\mathrm{O}$ acesso à EI fez diferença para as meninas nas três variáveis dependentes investigadas, ao passo que para os meninos o acesso à EI foi apenas associado a menos comportamentos internalizantes no EF.

As diferenças por sexo encontradas sugerem maior influência positiva da EI sobre as meninas. A esse respeito, há indícios de que as professoras da EI avaliam mais positivamente as meninas, seja quanto ao comportamento ou ao desempenho (Gardinal \& Marturano, 2007), e também avaliam sua relação com as meninas como mais próxima (Howes, Phillipsen, \& Peisner-Feinberg, 2000). Essa experiência precoce mais positiva na EI pode, em parte, explicar o efeito favorável mais generalizado da EI sobre as meninas e se explica, por sua vez, pela influência da socialização na família, que prepara melhor as meninas que os meninos para se adaptarem à escola, um contexto tipicamente "feminino" (Orr, 2011).

\section{Conclusão}

A pesquisa mostrou a importância da EI, que influencia o desenvolvimento de habilidades sociais, de modo mais intenso nas meninas que nos meninos. Mostrou também que este efeito não é compensado depois, com a experiência de escolaridade no EF.

Cabe apontar algumas limitações do estudo. Ele se baseou só no julgamento do professor. Informações dos colegas de turma contribuiriam para ampliar a compreensão das questões investigadas, particularmente sobre a competência social. Quanto ao tratamento estatístico, o modelo adotado não permitiu reconstituir diferentes cursos de desenvolvimento nem relações transacionais entre domínios. O estudo também não levou em conta variáveis contemporâneas com influência nos resultados. Fatores relacionados à experiência atual das crianças, como qualidade da escola de EF, relação professor-aluno e rede de relações com os pares poderiam atuar como moderadores do impacto da educação infantil sobre a competência interpessoal e o ajustamento psicossocial apresentados pelos alunos. A utilização de modelos de regressão e análise de pistas permitiria explorar relações complexas entre variáveis, que este estudo não contemplou.

Isso posto, cabe destacar qualidades que conferem peso aos resultados. Seguindo por três anos um número significativo de alunos de escolas públicas, a pesquisa gerou resultados sugestivos de um impacto positivo duradouro da educação infantil no curso do desenvolvimento psicossocial das crianças, controlada a sua origem socioeconômica. Reitera-se assim a importância de garantir para todas as crianças o acesso à educação infantil. Já os resultados que não mostram diferenças entre crianças com um ou dois anos de EI demandam maior investigação, incluindo outras variáveis que poderiam influenciar os resultados, seja no âmbito escolar ou familiar. Outras questões levantadas, demandando aprofundamento, dizem respeito ao curso de desenvolvimento das habilidades sociais, ao efeito protetor cumulativo dessas habilidades e ao impacto diferencial da educação infantil em meninos e meninas.

\section{Referências}

Achenbach, T. M., \& Edelbrock, C. S. (1979). The child behavior profile: II. Boys aged 12-16 and girls aged 6-11 and 12-16. Journal of Consulting and Clinical Psychology, 47(2), 223-233.

Associação Brasileira de Empresas de Pesquisa. (2003). Critério de classificação econômica Brasil. Dados com base no levantamento socioeconômico, IBOPE, 2000. Recuperado em 14 de fevereiro, 2007, de http://www.abep.org.br

Bandeira, M., Del Prette, Z. A. P., Del Prette, A., \& Magalhães, T. (2009). Validação das escalas de habilidades sociais, comportamentos problemáticos e competência acadêmica (SSRS-BR) para o ensino fundamental. Psicologia: Teoria e Pesquisa, 25(2), 271-282.

Bandeira, M., Rocha, S. S., Freitas, L. C., Del Prette, Z. A. P., \& Del Prette, A. (2006). Habilidades sociais e variáveis sociodemográficas em estudantes do ensino fundamental. Psicologia em Estudo, 11(3), 541-549.

Bee, H. (2003). A criança em desenvolvimento (9. ed). Porto Alegre, RS: Artmed.

Berry, D., \& O'Connor, E. (2010). Behavioral risk, teacher-child relationships, and social skill development across middle childhood: A child-by-environment analysis of change. Journal of Applied Developmental Psychology, 31(1), 1-14.

Blandon, A. Y., Calkins, S. D., Grimm, K. J., Keane, S. P., \& O'Brien, M. (2010). Testing a developmental cascade model of emotional and social competence and early peer acceptance. Development and Psychopathology, 22(4), 737-748. 
Pizato, E. C. G., Marturano, E. M. \& Fontaine, A. M. G. V. (2014). Trajetórias de Habilidades Sociais e Problemas de Comportamento no Ensino Fundamental: Influência da Educação Infantil.

Bohlin, G., \& Hagekull, B. (2009). Socio-emotional development: From infancy to young adulthood. Scandinavian Journal of Psychology, 50(6), 592-601.

Bolsoni-Silva, A. T., Marturano, E. M., \& Freiria, R. L. B. (2010). Indicativos de problemas de comportamento e de habilidades sociais em crianças: Um estudo longitudinal. Psicologia: Reflexão e Crítica, 23, 506-515.

Bornstein, M. H., Hahn, C.-S., \& Haynes, O. M. (2010). Social competence, externalizing, and internalizing behavioral adjustment from early childhood through early adolescence: Developmental cascades. Development and Psychopathology, 22(4), 717-735.

Brownell, C., Belsky, J., Booth-LaForce, C., Bradley, R., Campbell, S. B., Clarke-Stewart, K. A., ...Cox, M. (2008). Social competence with peers in third grade: Associations with earlier peer experiences in childcare. Social Development, 17, 419-453.

Campos, M. M., Esposito, Y. L., Bhering, E., Gimenes, N., \& Abuchaim, B. (2011). A qualidade da educação infantil: Um estudo em seis capitais brasileiras. Cadernos de Pesquisa, 41, 21-54.

Chen, X., \& French, D. C. (2008). Children's social competence in cultural context. Annual Review of Psychology, 59, 591616.

Côté, S., Vaillancourt, T., Barker, E. D., Nagin, D., \& Tremblay, R. E. (2007). The joint development of physical and indirect aggression: Predictors of continuity and change during childhood. Development and Psychopathology, 19, 37-55.

Dearing, E., McCartney, K., \& Taylor, B. A. (2006). Withinchild associations between family income and externalizing and internalizing problems. Developmental Psychology, 42(2), 237-252.

Del Prette, Z. A. P., \& Del Prette, A. (2005). Psicologia das habilidades sociais na infância: Teoria e prática. Petrópolis, RJ: Vozes.

Fanti, K. A., \& Henrich, C. C. (2010). Trajectories of pure and co-occurring internalizing and externalizing problems from age 2 to age 12: Findings from the National Institute of Child Health and Human Development Study of Early Child Care. Developmental Psychology, 46(5), 1159-1175.

Gardinal, E. C., \& Marturano, E. M. (2007). Meninos e meninas na educação infantil: Associação entre comportamento e desempenho. Psicologia em Estudo, 12, 541-551.

Gazelle, H., \& Ladd, G. W. (2003). Anxious solitude and peer exclusion: A diathesis stress model of internalizing trajectories in childhood. Child Development, 74, 257-278.

Howes, C., Phillipsen, L. C., \& Peisner-Feinberg, E. (2000). The consistency of perceived teacher-child relationships between preschool and kindergarten. Journal of School Psychology, 38, 113-132.

Kettler, R. J., Elliott, S. N., Davies, M., \& Griffin, P. (2011). Testing a multi-stage screening system: Predicting performance on Australia's national achievement test using teachers' ratings of academic and social behaviors. School Psychology International, 33, 93-111.

Marturano, E. M., Trivelatto-Ferreira, M. C., \& Gardinal, E. C. (2009). Estresse cotidiano na transição da $1^{\mathrm{a}}$ série: Percepção dos alunos e associação com desempenho e ajustamento. Psicologia Reflexão e Crítica, 22(1), 93-101.

Miner, J. L., \& Clarke-Stewart, K. A. (2008). Trajectories of externalizing behavior from age 2 to age 9: Relations with gender, temperament, ethnicity, parenting, and rater. Developmental Psychology, 44(3), 771-786.
Morgan, P. L., Farkas, G., \& Wu, Q. (2009). Kindergarten predictors of recurring externalizing and internalizing psychopathology in the third and fifth grades. Journal of Emotional and Behavioral Disorders, 17(2), 67-79.

Orr, A. J. (2011). Gendered capital: Childhood socialization and the "boy crisis" in education. Sex Roles, 65, 271-284.

Pereira, M. T., Marturano, E. M., Gardinal-Pizato, E. C., \& Fontaine, A. M. G. (2011). Possíveis contribuições da educação infantil no desempenho e competência social de escolares. Psicologia Escolar e Educacional, 15, 101-109.

Reynolds, A. J., \& Temple, J. A. (2008). Cost-effective early childhood development programs from preschool to third grade. Annual Review of Clinical Psychology, 9, 109-139.

Reynolds, M. R., Sander, J. B., \& Irvin, M. J. (2010). Latent curve modeling of internalizing behaviors and interpersonal skills through elementary school. School Psychology Quarterly, 25, 189-201.

Robbers, S. C. C., Bartels, M., van Oort, F. V. A., van Beijsterveldt, C. E. M., van der Ende, J., Verhulst, F. C., ... Huizink, A. C. (2010). A twin-singleton comparison of developmental trajectories of externalizing and internalizing problems in 6- to 12-year-old children. Twin Research and Human Genetics, 13, 79-87.

Sallquist, J. V., Eisenberg, N., Spinrad, T. L., Reiser, M., Hofer, C., Zhou, Q., ...Eggun, N. (2009). Positive and negative emotionality: Trajectories across six years and relations with social competence. Emotion, 9, 15-28.

Silver, R. B., Measelle, J. R., Armstrong, J. M., \& Essex, M. J. (2010). The impact of parents, child care providers, teachers, and peers on early externalizing trajectories. Journal of School Psychology, 48(6), 555-583.

Skinner, E. A., \& Zimmer-Gembeck, M. J. (2007). The development of coping. Annual Review of Psychology, 58, 119-144.

Sylva, K., Melhuish, E., Sammons, P., Siraj-Blatchford, I., \& Taggart, B. (2004). The Effective provision of pre-school education (EPPE) project: Findings from the early primary years. London: Institute of Education, University of London. Retrieved November, 2011, from www.ioe.ac.uk/school/ ecpe/eppe/

Trivellato-Ferreira, M. C., \& Marturano, E. M. M. (2008). Recursos da criança, da família e da escola predizem competência na transição da $1^{\mathrm{a}}$ série. Revista Interamericana de Psicologia, 42, 407-410.

Vieira, L. M. F. (2010). A Educação Infantil e o Plano Nacional de Educação: As propostas da CONAE 2010. Educação \& Sociedade, 31(112), 809-831. 